PHYSICAL REVIEW D 94, 094007 (2016)

\title{
Magnetic field effects on the static quark potential at zero and finite temperature
}

\author{
Claudio Bonati, ${ }^{*}$ Massimo D’Elia, ${ }^{\dagger}$ Marco Mariti, ${ }^{\ddagger}$ Michele Mesiti, ${ }^{\S}$ Francesco Negro, ${ }^{\|}$and Andrea Rucci ${ }^{\circ}$ \\ Dipartimento di Fisica dell'Università di Pisa and INFN_Sezione di Pisa, \\ Largo Pontecorvo 3, I-56127 Pisa, Italy
}

\author{
Francesco Sanfilippo ** $^{* *}$ \\ School of Physics and Astronomy, University of Southampton, SO17 1BJ Southampton, United Kingdom
} (Received 5 August 2016; published 10 November 2016)

\begin{abstract}
We investigate the static $Q \bar{Q}$ potential at zero and finite temperature in the presence of a constant and uniform external magnetic field $\vec{B}$, for several values of the lattice spacing and for different orientations with respect to $\vec{B}$. As a byproduct, we provide continuum limit extrapolated results for the string tension, the Coulomb coupling and the Sommer parameter at $T=0$ and $B=0$. We confirm the presence in the continuum of a $B$-induced anisotropy, regarding essentially the string tension, for which it is of the order of $15 \%$ at $|e| B \sim 1 \mathrm{GeV}^{2}$ and would suggest, if extrapolated to larger fields, a vanishing string tension along the magnetic field for $|e| B \gtrsim 4 \mathrm{GeV}^{2}$. The angular dependence for $|e| B \lesssim 1 \mathrm{GeV}^{2}$ can be nicely parametrized by the first allowed term in an angular Fourier expansion, corresponding to a quadrupole deformation. Finally, for $T \neq 0$, the main effect of the magnetic field is a general suppression of the string tension, leading to a early loss of the confining properties: this happens even before the appearance of inverse magnetic catalysis in the chiral condensate, supporting the idea that the influence of the magnetic field on the confining properties is the leading effect originating the decrease of $T_{c}$ as a function of $B$.
\end{abstract}

DOI: $10.1103 /$ PhysRevD.94.094007

\section{INTRODUCTION}

In the Standard Model, the strong and the electroweak sectors are connected by quarks, which are subject to both type of interactions. In general, electroweak interactions are expected to induce relatively small corrections to strong interaction dynamics; however, this may be not true in the presence of very strong electromagnetic backgrounds, with field values comparable to the QCD scale. This is a situation which is relevant to many contexts, ranging from noncentral heavy ion collisions [1-6] and the cosmological electroweak phase transition [7,8], with magnetic fields reaching or exceeding $10^{16} \mathrm{~T}\left(e B \sim 1 \mathrm{GeV}^{2}\right)$, to magnetars [9], where magnetic fields are expected to be of the order of $10^{11} \mathrm{~T}$ on the surface but could be significantly larger in the inner cores.

How strong interactions get modified by such large magnetic fields has been the subject of many recent theoretical studies (see, e.g., Refs. [10,11] for reviews), with lattice simulations representing a viable and effective tool to explore the issue starting from the first principles of QCD. One important feature is that gluon fields, even if not

\footnotetext{
*claudio.bonati@df.unipi.it

†massimo.delia@unipi.it

*mariti@df.unipi.it

\$michele.mesiti@pi.infn.it

"fnegro@pi.infn.it

Tandrea.rucci@pi.infn.it

***sanfilippo@soton.ac.uk
}

directly coupled to electromagnetic fields, undergo significant modifications, through effective QED-QCD interactions induced by quark loops, as can be seen both by lattice simulations [12-28] and by analytical studies using several approaches, which range from perturbation theory to effective field theories, and from Nambu-Jona-Lasinio models to functional renormalization group and holographic techniques [29-52]. A striking consequence of the QEDQCD coupling is the distortion of the zero temperature static quark-antiquark potential. Results reported in Ref. [21] showed the emergence of anisotropies both in the linear part (string tension) and in the Coulomb part of the potential. This behavior is consistent with the results of some of the existing model computations [53-57] and may have relevant phenomenological consequences, especially for the spectrum of heavy quark bound states [58-67].

The purpose of this study is to move one step forward in our comprehension of magnetic-induced effects on nonperturbative QCD dynamics. First of all, we present a refinement of our zero temperature data following three different directions:

(i) A complete analysis of the angular dependence of the potential (in Ref. [21] only quark-antiquark separations parallel and orthogonal to the magnetic field were analyzed). This is important to allow for a realistic modeling of the quark-antiquark potentials to be used in the computation of the spectrum of heavy quarkonia. 
(ii) The inclusion of new simulations on finer lattices will permit us to extrapolate results reported in Ref. [21] to the continuum limit.

(iii) The investigation of a regime of fields significantly larger than those used in Ref. [21], in order to inquire whether new unexpected phenomena may take place in the QCD vacuum under the influence of extremely strong background fields.

As a byproduct of this investigation, we will also provide a continuum extrapolation of the static quark-antiquark potential, in the usual Cornell parametrization, with physical quark masses and for the standard case of vanishing magnetic field.

In the second part of our study, we investigate how the effect of the magnetic field on the static potential gets modified by the temperature, a step which is important for a full comprehension of the properties of the thermal medium in the presence of magnetic backgrounds. In this case the static potential (more correctly the free energy) is extracted from Polyakov loop correlators in place of Wilson loop expectation values used at $T=0$. For temperatures below the pseudocritical temperature $T_{c} \sim 155 \mathrm{MeV}$ (at which chiral symmetry gets restored and quark and gluon degrees of freedom deconfine), one still expects that heavy quark-antiquark interactions can be described in terms of a confining potential, with a string tension which goes to zero as $T_{c}$ is approached. Two main questions will be addressed by our study in this regime: (i) does the anisotropy survive also in the finite temperature case? (ii) does the magnetic field enhance the suppression of the string tension, meaning that a phenomenon similar to inverse magnetic catalysis [17], observed for chiral symmetry, takes place also for the confining properties of the medium?

The paper is organized as follows. In Sec. II we illustrate the setup of our numerical simulations and the techniques adopted to extract the static potential, both at zero and at finite temperature, in Sec. III we present our numerical results and finally, in Sec. IV, we draw our conclusions.

\section{NUMERICAL METHODS}

The discretization of the $N_{f}=2+1$ QCD action adopted in this work is a combination of the Symanzik tree-level improved gauge action and of stout improved rooted staggered fermions. Explicitly, the partition function is written as

$$
Z(B)=\int \mathcal{D} U e^{-\mathcal{S}_{\mathrm{YM}}} \prod_{f=u, d, s} \operatorname{det}\left(D_{\mathrm{st}}^{f}[B]\right)^{1 / 4} .
$$

Here $\mathcal{D} U$ is the functional integration over the $S U(3)$ link variables and $\mathcal{S}_{\mathrm{YM}}$ stands for the tree-level improved action $[68,69]$,

$$
\mathcal{S}_{\mathrm{YM}}=-\frac{\beta}{3} \sum_{i, \mu \neq \nu}\left(\frac{5}{6} W_{i ; \mu \nu}^{1 \times 1}-\frac{1}{12} W_{i ; \mu \nu}^{1 \times 2}\right),
$$

where the real part of the trace of the $1 \times 1$ and $1 \times 2$ loops is denoted by $W_{i ; \mu \nu}^{1 \times 1}$ and $W_{i ; \mu \nu}^{1 \times 2}$ respectively. Finally, the staggered Dirac matrix

$$
\begin{aligned}
\left(D_{\mathrm{st}}^{f}\right)_{i, j}= & a m_{f} \delta_{i, j}+\sum_{\nu=1}^{4} \frac{\eta_{i ; \nu}}{2}\left(u_{i ; \nu}^{f} U_{i ; \nu}^{(2)} \delta_{i, j-\hat{\nu}}\right. \\
& \left.-u_{i-\hat{\nu} ; \nu}^{f *} U_{i-\hat{\nu} ; \nu}^{(2) \dagger} \delta_{i, j+\hat{\nu}}\right)
\end{aligned}
$$

is written by using the two-time stout-smeared links $U_{i ; \mu}^{(2)}$ [70] (with isotropic smearing parameter $\rho=0.15$ ) and the $U(1)$ parallel transporters $u_{i ; \mu}^{f}$, where the $i$ index denotes the position in the lattice and the $\mu$ index denotes the direction of the link.

For a magnetic field directed along the $\hat{z}$ direction, a possible choice of the Abelian transporters is $\left(q_{f}\right.$ is the fermion charge)

$$
u_{i ; y}^{f}=e^{i a^{2} q_{f} B_{z} i_{x}},\left.\quad u_{i ; x}^{f}\right|_{i_{x}=L_{x}}=e^{-i a^{2} q_{f} L_{x} B_{z} i_{y}},
$$

with all the other $U(1)$ link variables being equal to 1 . Moreover, for these transporters to describe a uniform magnetic field on the lattice torus, the value of $B_{z}$ has to satisfy the following quantization condition [71-74]:

$$
e B_{z}=6 \pi b_{z} /\left(a^{2} N_{x} N_{y}\right) ; \quad b_{z} \in \mathbb{Z} .
$$

In the following we will consider also the case of a magnetic field $\vec{B}$ not directed along one of the coordinate axes. In this case, each component of $\vec{B}$ generates transporters analogous to Eq. (3) in the corresponding orthogonal plane, and the final $U(1)$ phases appearing in the fermion matrix are the product of the phases that would be generated by each component separately. All the components have to satisfy a quantization condition analogous to Eq. (4): as a consequence, the magnetic field on the lattice can be represented by the vector $\vec{b}$ having integer components. If $N_{x}=N_{y}=N_{z}$, i.e. if the quantization condition in Eq. (4) is the same for all components, then the magnetic field $\vec{B}$ is parallel to $\vec{b}$.

The values of the bare parameters used in our simulations have been chosen so as to move on a line of constant physics, corresponding to physical values of the quark masses: to do that, we have followed the determination reported in Refs. [75,76], based also on the fact that the magnetic field does not alter the value of the lattice spacing [15]. In Table I we list for convenience the values of the bare parameters adopted in the zero temperature runs: some entries refer also to simulations already reported in 
TABLE I. Simulation parameters for the $T=0$ runs, chosen according to Refs. $[75,76]$ and corresponding to physical values of the pion mass and of the strange-to-light mass ratio, $m_{s} / m_{u, d}=28.15$. The systematic error on $a$ is about 2\%-3\% [76,77].

\begin{tabular}{lcccc}
\hline \hline Lattice size & $a(\mathrm{fm})$ & $\beta$ & $a m_{s}$ & $b$ \\
\hline $24^{4}$ & $0.2173(4)$ & 3.55 & 0.1020 & $0,12,16,24,32,40$ \\
$32^{4}$ & $0.1535(3)$ & 3.67 & 0.0639 & $0,12,16,24,32,40$ \\
$40^{4}$ & $0.1249(3)$ & 3.75 & 0.0503 & $0,8,12,16,24,32,40$ \\
$48^{3} \times 96$ & $0.0989(2)$ & 3.85 & 0.0394 & $0,8,16,24,32$ \\
\hline \hline
\end{tabular}

Ref. [21]; most of the new runs have been performed on the finest $48^{3} \times 96$ lattice.

The Rational Hybrid Monte Carlo [78-80] algorithm has been used to sample gauge configurations. To determine the interquark potential in the confined phase, statistics of $O\left(10^{3}\right)-O\left(10^{4}\right)$ trajectories have been collected for each value of the magnetic background, with measures of Wilson loops performed every 5 trajectories.

In order to reduce the UV noise, we used HYP [81] and APE [82] smearing in the following combination: one step of HYP smearing for temporal links (with the parameters of the HYP2-action reported in Ref. [83]) and a variable number of isotropic APE smearing steps (with parameter $\alpha_{\mathrm{APE}}=0.25$ ), which has been chosen large enough to significantly reduce the noise, but still small enough to introduce no significant systematic effects. In practice, we verified that a number of steps between 20 and 40 (depending on the lattice spacing) satisfies these requirements. The potential was extracted from planar Wilson loops of size $\vec{n} \times n_{t}$, making use of the definition

$$
\begin{aligned}
a V_{\mathrm{eff}}\left(a \vec{n}, a n_{t}\right) & \equiv \log \left(\frac{\left\langle W\left(\vec{n}, n_{t}\right)\right\rangle}{\left\langle W\left(\vec{n}, n_{t}+1\right)\right\rangle}\right), \\
a V(a \vec{n}) & =\lim _{n_{t} \rightarrow \infty} a V_{\mathrm{eff}}\left(a \vec{n}, a n_{t}\right),
\end{aligned}
$$

i.e. by finding, for each fixed $\vec{n}$, a range of $n_{t}$ values where the rhs of the previous definition is stable, in order to perform a fit to a constant. The fit range has been varied in order to estimate the systematic error associated with its choice. It is important to stress that since rotation symmetry is explicitly broken by the external field, different orientations of the Wilson loop have to be studied independently in order to determine properly the potential.

As an example of the procedure described above, in Fig. 1 we report the values $a V_{\text {eff }}\left(a \vec{n}, a n_{t}\right)$ as a function of $n_{t}$, obtained on the finest $48^{3} \times 96$ lattice with a magnetic background corresponding to $\vec{b}=32 \hat{z}$ (|e $\left.\mid B \sim 1 \mathrm{GeV}^{2}\right)$. Data refer to the case $|\vec{n}|=3$ and we report separately results obtained along the directions parallel or transverse to $\vec{B}$, for two different APE smearing levels.

For finite temperature simulations, the static quarkantiquark potential has been determined from Polyakov loop correlators

$$
C(\vec{n}, T)=\left\langle\operatorname{Tr} L(\vec{r}) \operatorname{Tr} L^{\dagger}(\vec{r}+\vec{n})\right\rangle
$$

where $\vec{r}$ and $\vec{n}$ are dimensionless lattice vectors. This observable is related to the free energy $F_{Q \bar{Q}}(a \vec{n}, T)$ of a static quark-antiquark pair separated by a distance $a \vec{n}$. In principle, at a perturbative level, such correlator takes contribution from both the singlet and octet color channels [84-87]; however, one can show that only the singlet contribution survives in the correlator in Eq. (6) $[88,89]$, so that one can consistently define $C(\vec{n}, T) \propto \exp \left(-F_{Q \bar{Q}}(a \vec{n}\right.$, $T) / T$ ), where $F_{Q \bar{Q}}$ is the free energy of the static pair in the singlet channel. Therefore, we shall adopt the definition

$$
F_{Q \bar{Q}}(a \vec{n}, T)=-\frac{1}{a N_{t}} \log C(\vec{n}, T)
$$

which, apart from temperature dependent additive renormalizations, we take as an estimate of the static quark-antiquark potential at finite temperature $\left[N_{t}\right.$ is the temporal size of the lattice, which is related to the temperature of the system by $T=\left(a N_{t}\right)^{-1}$ ].

For the finite temperature runs, we have adopted statistics comparable to $T=0$ runs [i.e. $O\left(10^{3}\right)-O\left(10^{4}\right)$ trajectories for each run], with measures of the Polyakov loop correlators

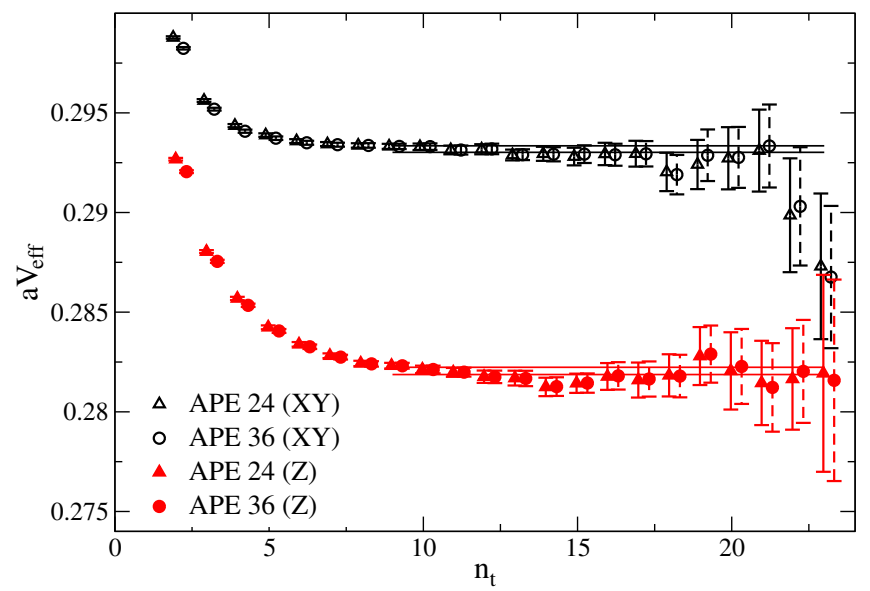

FIG. 1. Wilson loop combination $a V_{\text {eff }}\left(a \vec{n}, a n_{t}\right)$ defined in Eq. (5) for $|\vec{n}|=3$ as a function of $n_{t}$. Results refer to two different values of the APE smearing level and to different orientations (orthogonal, $X Y$, or parallel, $Z$ ) of the quarkantiquark separation relative to $\vec{B}=B \hat{z}$. The simulation has been performed on a $48^{3} \times 96$ lattice at $|e| B \simeq 1 \mathrm{GeV}^{2}$. 
performed after each trajectory and one step of HYP smearing in the temporal direction (with the same parameters as for the $T=0$ case) to suppress the UV fluctuations.

\section{RESULTS}

In this section we present the results of our simulations. We start with a determination of the static potential at $T=0$ and $B=0$ for which, using results at four different lattice spacings, we are able to obtain a reliable continuum extrapolation. We then move to the results obtained for $B \neq 0$ : we investigate the angular dependence of the anisotropic static potential, which is then continuum extrapolated using the numerical data for quark-antiquark separations orthogonal or parallel to the external magnetic field. We point out some interesting features that seem to emerge in the limit of very large magnetic field and finally we discuss the modifications induced by a nonvanishing temperature.

\section{A. $T=0, B=0$ : Continuum extrapolated results}

The zero temperature static quark-antiquark potential has been largely investigated by means of phenomenological studies and lattice simulations and it has been shown to be well described by the so-called Cornell potential [90]

$$
V(r)=-\frac{\alpha}{r}+\sigma r+V_{0},
$$

where $\sigma$ is the string tension, $\alpha$ is the Coulomb parameter and $V_{0}$ is an arbitrary constant term. A related quantity that is often convenient to introduce is the so-called Sommer parameter $r_{0}$, which is defined by the equation [91]

$$
\left.r_{0}^{2} \frac{d V}{d r}\right|_{r_{0}}=1.65
$$

This parameter can be related to those entering Eq. (8) by

$$
r_{0}=\sqrt{\frac{1.65-\alpha}{\sigma}}
$$

so that only two out of $r_{0}, \alpha$ and $\sigma$ are independent quantities.

In Fig. 2 we show the results obtained for the potential, using the procedure outlined in Sec. II, for different lattice spacings. In this case, since no magnetic background is present, Wilson loops have been averaged over the different spatial directions. For each lattice spacing, data have been fitted according to Eq. (8), in order to extract the values of $\sigma, \alpha$ and $r_{0}\left(\sigma\right.$ and $r_{0}$ have been used as independent fit parameters). In all cases the fit turned out to have a reduced chi-square around 1 and we verified the stability of the results against modifications of the fit range adopted to extract the parameters.

The values of $\sigma, r_{0}$ and $\alpha$ obtained at each lattice spacing are shown in Fig. 3: they will be used in Sec. III C as reference values to determine the anisotropies induced by

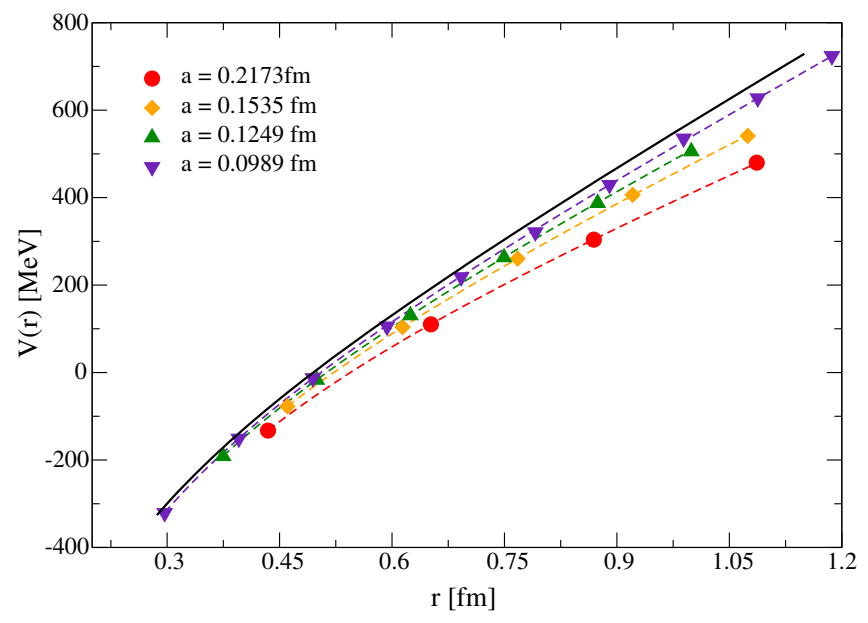

FIG. 2. Static potential as a function of the quark-antiquark distance in physical units and for various lattice spacings. Dashed curves represent the result of a fit according to the Cornell potential in Eq. (8), while the solid curve represents the continuum extrapolation. The constant $V_{0}$ has been shifted, for each lattice spacing separately, so that the potential vanishes at $r_{0}$.

the presence of the magnetic field, while in the following we discuss their extrapolation to the continuum limit.

If we take into account only the three smallest lattice spacings, the continuum extrapolation can be performed in all cases by using just the expected leading-order $a^{2}$ scaling: results are reported in Table II. ${ }^{1}$ We also explored the possibility of fitting data at all lattice spacings, but taking into account also $O\left(a^{4}\right)$ corrections: the continuum results obtained in this way are in good agreement with the ones we got previously using only the leading $a^{2}$ dependence, and the difference between the two values is used as an estimate of the systematic error related to the continuum extrapolation, which is reported in Table II as well (see also Fig. 3). Values obtained for $r_{0}$ and $\sigma$ are in good agreement with phenomenological estimates and with previous lattice determinations [92-98] (see also Sec. 9.2 of Ref. [99] for a recent review).

\section{B. $T=0, B \neq 0$ : Angular dependence}

The static potential studied in the previous subsection is isotropic, i.e. it depends only on the modulus $r$ of the distance between the quark and the antiquark. In Ref. [21] it was shown that, at least at nonvanishing lattice spacing, this property is lost in the presence of a magnetic background which explicitly breaks rotational invariance. The investigation carried out in Ref. [21] was restricted to the cases of quark-antiquark separations parallel or orthogonal to the magnetic field, the final result of that work being that in both

\footnotetext{
${ }^{1}$ Such a linear scaling in $a^{2}$ is compatible with $\sigma$ and $r_{0}$ data also in the whole range of lattice spacings; however, to be conservative, in Table II we report the values obtained by fitting just the three finest lattices.
} 

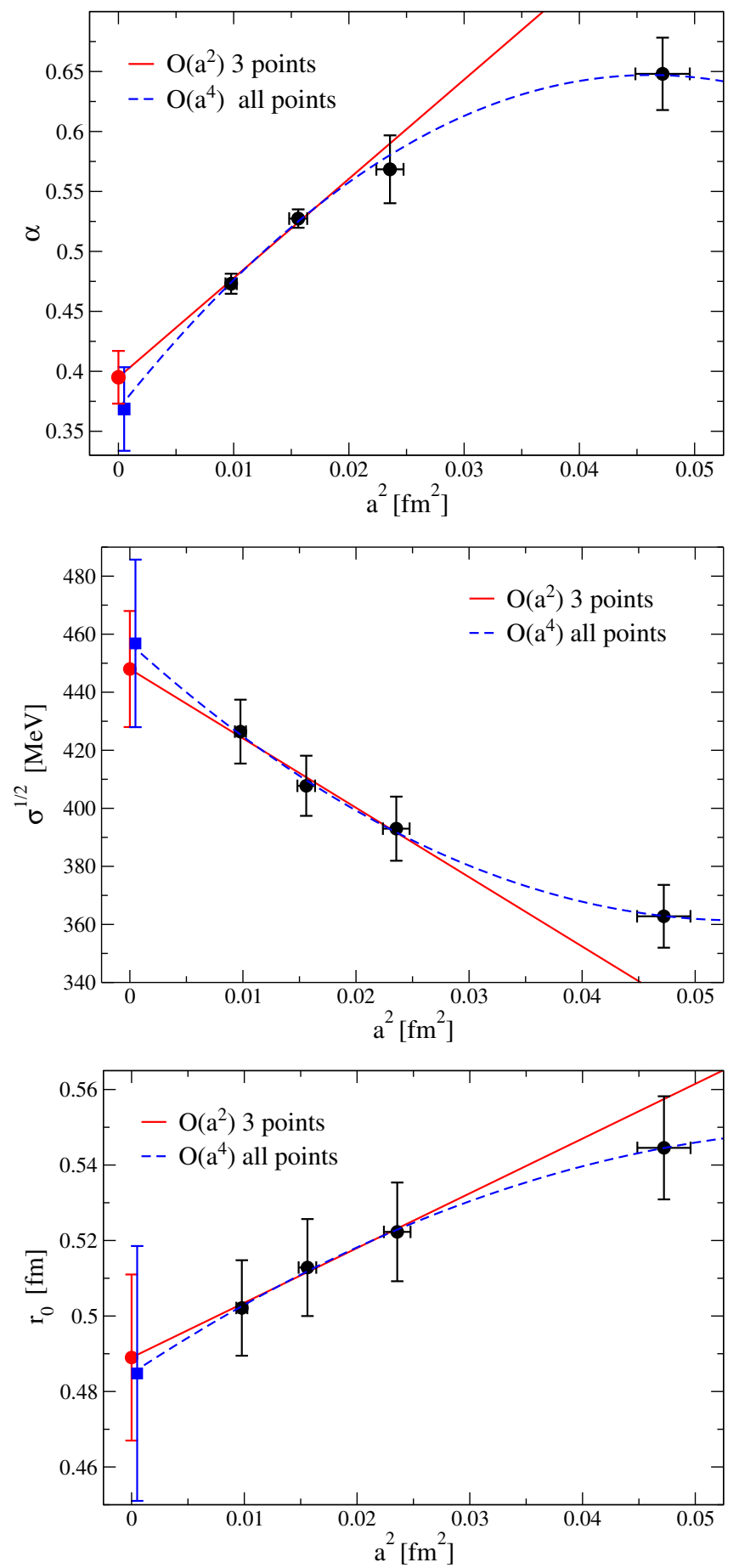

FIG. 3. Continuum extrapolation of the $Q \bar{Q}$ potential parameters $\alpha$ (top), $\sqrt{\sigma}$ (center) and $r_{0}$ (bottom) at $T=0$ and $B=0$. Two different extrapolations are shown: linear in $a^{2}$, using only data from the three smallest lattice spacings (red solid line), and including $a^{4}$ corrections on the whole range of lattice spacings (blue dashed line).

these directions the potential is still well described by Eq. (8), but the parameters depend on the direction. In particular, the string tension is larger (smaller) in the direction orthogonal (parallel) to $\vec{B}$, while $\alpha$ has an opposite behavior.
TABLE II. Continuum extrapolated results for $\alpha, \sqrt{\sigma}$ and $r_{0}$. We report both the statistical errors of the $O\left(a^{2}\right)$ fit performed by using the three smallest lattice spacings, and an estimate of the systematical error, obtained by comparing with the result of the $O\left(a^{4}\right)$ fit on the whole range of lattice spacings (see also Fig. 3).

\begin{tabular}{lrl}
\hline \hline$\alpha$ & $0.395(22)(26)$ & \\
$\sqrt{\sigma}$ & $448(20)(09)$ & $\mathrm{MeV}$ \\
$r_{0}$ & $0.489(20)(04)$ & $\mathrm{fm}$ \\
\hline \hline
\end{tabular}

One of the purposes of our present investigation is to give a full description of the static potential for $B \neq 0$. There are some general features of the angular dependence that can be fixed a priori: while a generic anisotropic potential is a function of the distance and of two angular variables, in the case of a uniform magnetic field we can still rely on the residual rotational symmetry around $\vec{B}$, thus reducing to a single angular variable dependence, i.e. $V(r, \theta)$, where $\theta$ is the angle between the quarkantiquark separation and $\vec{B}$. Furthermore, since one expects in general symmetry under inversion of $\vec{B}$, we require $V(r, \theta)=V(r, \pi-\theta)$. Motivated by the results of Ref. [21] we make the ansatz that for each fixed value of $\theta$ the Cornell description still holds, i.e. that

$$
V(r, \theta, B)=\sigma(\theta, B) r-\frac{\alpha(\theta, B)}{r}+V_{0}(\theta, B) .
$$

The validity of such an assumption for all values of $\theta$ can of course only be verified a posteriori. Notice that we assume that also the constant term $V_{0}$ in the Cornell potential can take an angular dependence.

The most general description of the angular dependence of the Cornell parameters can be given in terms of a Fourier series; for instance for the string tension we can write

$$
\sigma(\theta, B)=\bar{\sigma}(B)\left(1-\sum_{n \geq 1} c_{2 n}^{\sigma}(B) \cos (2 n \theta)\right)
$$

where only Fourier coefficients which respect the symmetry under $\theta \rightarrow \pi-\theta$ have been used. Our general parametrization will therefore be the following one:

$$
\begin{aligned}
V(r, \theta)= & -\frac{\bar{\alpha}(B)}{r}\left(1-\sum_{n \geq 1} c_{2 n}^{\alpha}(B) \cos (2 n \theta)\right) \\
& +\bar{\sigma}(B) r\left(1-\sum_{n \geq 1} c_{2 n}^{\sigma}(B) \cos (2 n \theta)\right) \\
& +\bar{V}_{0}(B)\left(1-\sum_{n \geq 1} c_{2 n}^{V_{0}}(B) \cos (2 n \theta)\right) .
\end{aligned}
$$

How many Fourier coefficients are required to reliably describe the actual potential can be decided only on the 
basis of numerical results, which we are going to expose and discuss in the following.

In order to study the complete angular dependence of the potential one could in principle proceed in two different ways, i.e. by rotating either the Wilson loop or the magnetic field. In the first case a rotation of the spatial side of the Wilson loop by small angles (i.e. by less than $\pi / 2$ ) would require a significant modification of the spatial path; in particular, new cusps appear, which significantly modify the renormalization factors and make the comparison of results at different angles and lattice spacings more involved. For this reason we choose to rotate the magnetic field, a choice that requires us, however, to perform new Monte Carlo simulations for different orientations of the magnetic field, since $\vec{B}$ enters directly into the probability distribution of gauge configurations. As a consequence we fully investigated just a limited set of angular orientations; however, as we will show, this is sufficient to make the picture clear enough.

In simulations with $\vec{B}$ not directed along any of the lattice axes, in the computation of ratios appearing in Eq. (5) we have considered separately the Wilson loop directed along the $X, Y$ and $Z$ directions, which in general correspond to three different values of $\theta$. Simulations have been performed for two lattice spacings only, namely $a=0.0989$ and $0.1535 \mathrm{fm}$, using lattices of size $48^{3} \times 96$ and $32^{4}$ respectively. In both cases we considered a single value of the modulus of the magnetic field, $|\vec{b}| \simeq 32$, corresponding $|e| B \sim 1 \mathrm{GeV}^{2}$, since the spatial physical size is consistently $a L_{s} \simeq 5 \mathrm{fm}$. We studied the following combinations of magnetic field quanta: $\left(b_{x}, b_{y}, b_{z}\right)=(0,0,32),(4,13,29)$ and $(9,18,25)$, which give access to 8 different values of the angle $\theta$.

In Fig. 4 we show the results obtained for the potential as a function of $r$ for different values of $\theta$ on the finest

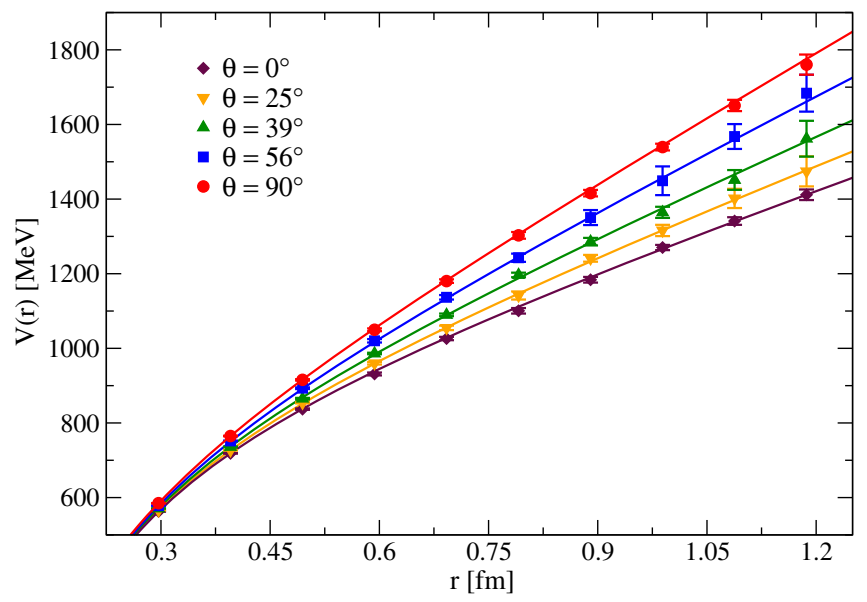

FIG. 4. The potential extracted using (5) from the $48^{3} \times 96$ lattice, for several values of the angle $\theta$ between the Wilson loop and $\vec{B}$ with $|e| B \simeq 1 \mathrm{GeV}^{2}$. No normalization has been performed on $V_{0}$ and best-fit curves are obtained using the form in Eq. (13).
TABLE III. Best-fit parameters for the potential in Eq. (13). The reduced chi-square values are $\chi^{2} / n_{\text {dof }}=53 / 74$ and $\chi^{2} / n_{\text {dof }}=36 / 34$ for the $48^{3} \times 96$ and the $32^{4}$ lattice respectively. Note that in this particular case the value of the chi-square is only a qualitative estimator of the fit goodness, since data are correlated (correlations have been propagated using a bootstrap procedure).

\begin{tabular}{lccc}
\hline \hline & $\bar{\alpha}$ & $\sqrt{\bar{\sigma}}(\mathrm{MeV})$ & $\bar{V}_{0}(\mathrm{MeV})$ \\
\hline $48^{3} \times 96$ & $0.493(6)$ & $414(2)$ & $644(5)$ \\
$32^{4}$ & $0.499(23)$ & $398(4)$ & $407(20)$ \\
\hline \hline & $c_{2}^{\alpha}$ & $c_{2}^{\sigma}$ & $c_{2}^{V_{0}}$ \\
\hline $48^{3} \times 96$ & $-0.130(10)$ & $0.262(7)$ & $-0.154(8)$ \\
$32^{4}$ & $-0.323(64)$ & $0.351(32)$ & $-0.428(52)$ \\
\hline \hline
\end{tabular}

$48^{3} \times 96$ lattice. A property of its angular dependence is clearly visible, which is present also for data on the coarser $32^{4}$ lattice and is in line with what observed in Ref. [21]: at fixed $r$, the potential increases as the angle between the quark-antiquark separation and $\vec{B}$ increases, and reaches its maximum in the plane orthogonal to $\vec{B}$.

A peculiar property that we found is that, as one tries to perform a best fit of data according to the ansatz given in Eq. (13), it is sufficient to include only the lowest-order Fourier coefficient $c_{2}$ for each parameter, in order to obtain a reasonable value of the reduced chi-square test (see the caption of Table III); if further coefficients are inserted in the fit, they come out to be compatible with zero within errors. The best-fit function obtained for the finer lattice is displayed in Fig. 4 as a function of $r$ for several $\theta$ values, while in Fig. 5 the same best-fit function is shown in a three-dimensional plot. The anisotropy of the potential is better seen by looking at the contour plot in Fig. 6. The best-fit parameters for both lattices are reported in Table III, and in Fig. 7 we show the angular variation of the string

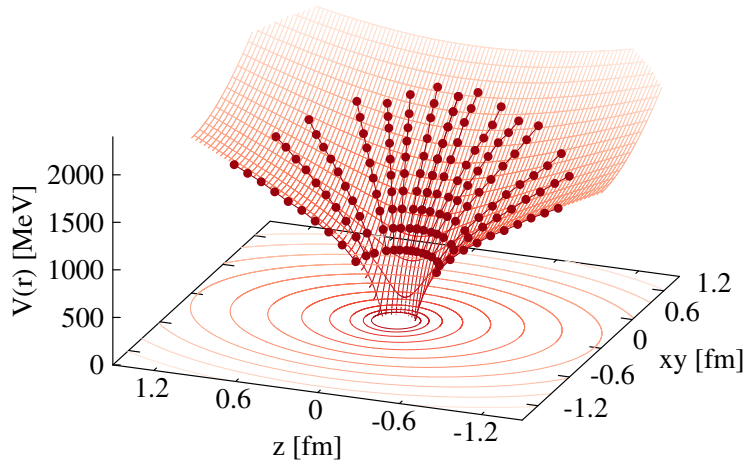

FIG. 5. A 3D plot of the potential as a function of the spatial coordinates. Data points refer to the $48^{3} \times 96$ lattice at $|e| B \sim$ $1 \mathrm{GeV}^{2}$ (with $\vec{B}$ directed along $Z$ ) and are fitted by the surface defined in Eq. (13). 


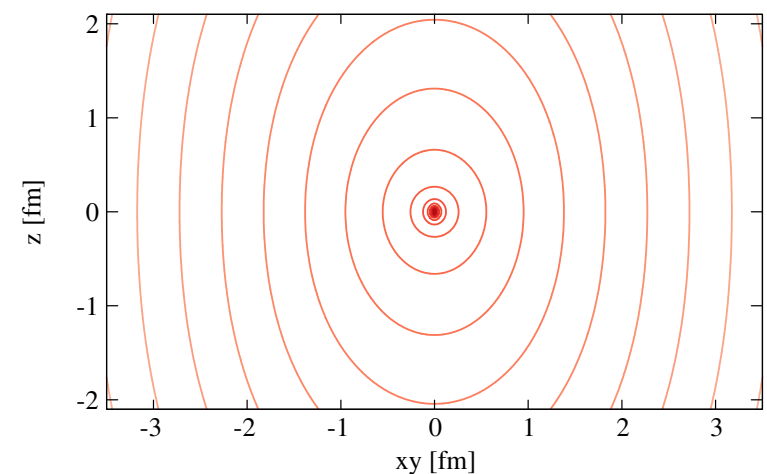

FIG. 6. Contour plot of the function displayed in Fig. 5. Contour lines are plotted every $500 \mathrm{MeV}$.

tension, from which it is clear that a single cosine term well describes data in both cases.

Another interesting feature emerges from the data in Table III: the constant term in the Cornell potential gets an angular dependence as well. This is a very peculiar feature, since that would imply an additive term in the potential which depends on $\theta$ but not on $r$. However, we stress that the associated Fourier coefficient $c_{2}^{V_{0}}$ is reduced by a factor of $\sim 2.8$ as one moves from the coarse to the fine lattice, consistently with the vanishing of such an angular dependence in the continuum limit. Notice that an analogous consideration, i.e. the vanishing of the angular dependence in the continuum limit, could be made for the Coulomb coupling, but not for the string tension; a more detailed discussion of this issue will be done in the following subsection. Finally, we stress that most of the $B$-dependence of the potential can be ascribed to Fourier coefficients, since the parameters $\bar{\alpha}, \bar{\sigma}$ and $\bar{V}_{0}$, which are sort of averaged values over different directions, show just a mild variation with respect to the $B=0$ case.

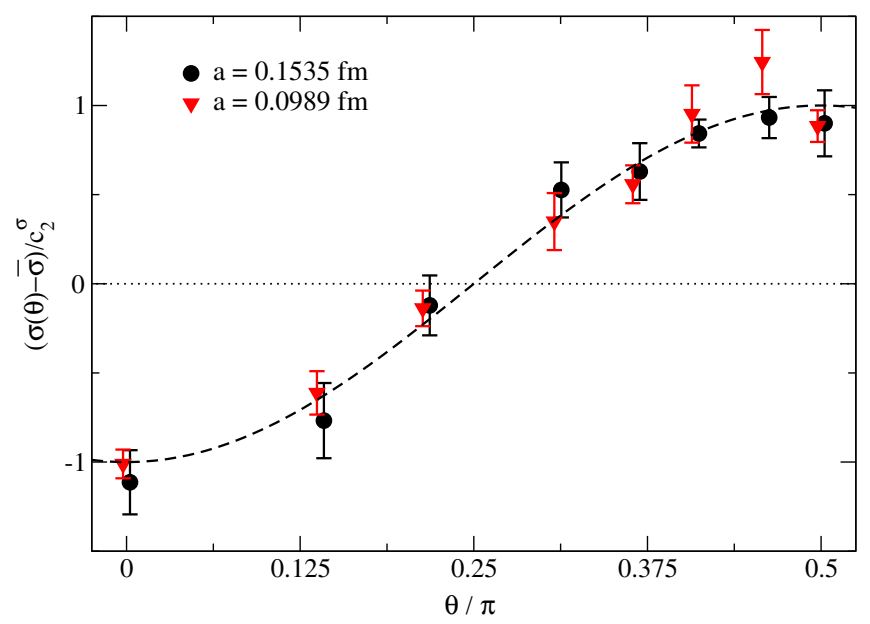

FIG. 7. Rescaled form of the string tension as function of the angle $\theta$ for the two lattices $32^{4}$ and $48^{3} \times 96$. The dashed line is the function $-\cos (2 \theta)$.
Having determined that the full angular dependence of the static quark potential can be described just by the first nontrivial harmonic term, we can determine all relevant parameters from an analysis of the potential along the directions parallel and orthogonal to $\vec{B}$, i.e. from $\theta=0$, $\pi / 2$, which are the standard quantities already explored in Ref. [21] and which have been extended in the present study to a finer lattice spacing and to different values of $B$.

\section{C. $T=0, B \neq 0$ : Continuum extrapolated results}

In this subsection, in order to perform a continuum extrapolation of the $B$-dependence of the static potential, we consider numerical results obtained at different values of the magnetic field and of the lattice spacing, for quarkantiquark separations parallel $(Z)$ or orthogonal $(X Y)$ to the magnetic field. That gives us access to the Cornell parameters in those directions, i.e. $\alpha_{X Y}$ and $\alpha_{Z}, \sigma_{X Y}$ and $\sigma_{Z}, V_{0, X Y}$ and $V_{0, Z}, r_{0, X Y}$ and $r_{0, Z}$. According to the analysis of the angular dependence given in the previous subsection, that provides us with enough information to characterize the complete behavior of the potential. Indeed, if for each parameter $\mathcal{O}$ we introduce its anisotropy

$$
\delta^{\mathcal{O}}(|e| B)=\frac{\mathcal{O}_{X Y}(|e| B)-\mathcal{O}_{Z}(|e| B)}{\mathcal{O}_{X Y}(|e| B)+\mathcal{O}_{Z}(|e| B)}
$$

and its average relative change with $B$

$$
R^{\mathcal{O}}(|e| B)=\frac{\mathcal{O}_{X Y}(|e| B)+\mathcal{O}_{Z}(|e| B)}{2 \mathcal{O}(|e| B=0)}
$$

we have, assuming that $c_{2 n}^{\mathcal{O}} \simeq 0$ when $n>1$,

$$
\delta^{\mathcal{O}}=\sum_{n} c_{2 n}^{\mathcal{O}} \simeq c_{2}^{\mathcal{O}}
$$

and

$$
\begin{aligned}
R^{\mathcal{O}}(|e| B)= & \frac{\overline{\mathcal{O}}(|e| B)}{\mathcal{O}(|e| B=0)}\left(1-\sum_{n \text { even }} c_{2 n}^{\mathcal{O}}\right) \\
& \simeq \frac{\overline{\mathcal{O}}(|e| B)}{\mathcal{O}(|e| B=0)}
\end{aligned}
$$

That is, such quantities (together with the values at $B=0$ ) are enough to fix all the coefficients giving a nontrivial contribution to Eq. (13) in the physically realized case, in which $c_{2 n} \simeq 0$ for $n>1$.

Results obtained in this way for the $c_{2}$ coefficients at the various magnetic fields and lattice spacings are shown in Fig. 8, while in Fig. 9 we report results for the quantities $R^{\mathcal{O}}(|e| B)$. A remarkable feature is that these quantities show a very mild variation with the magnetic field, so that most of the dependence of the potential on $B$ can be ascribed to the anisotropy coefficients $c_{2}$. Moreover, the 

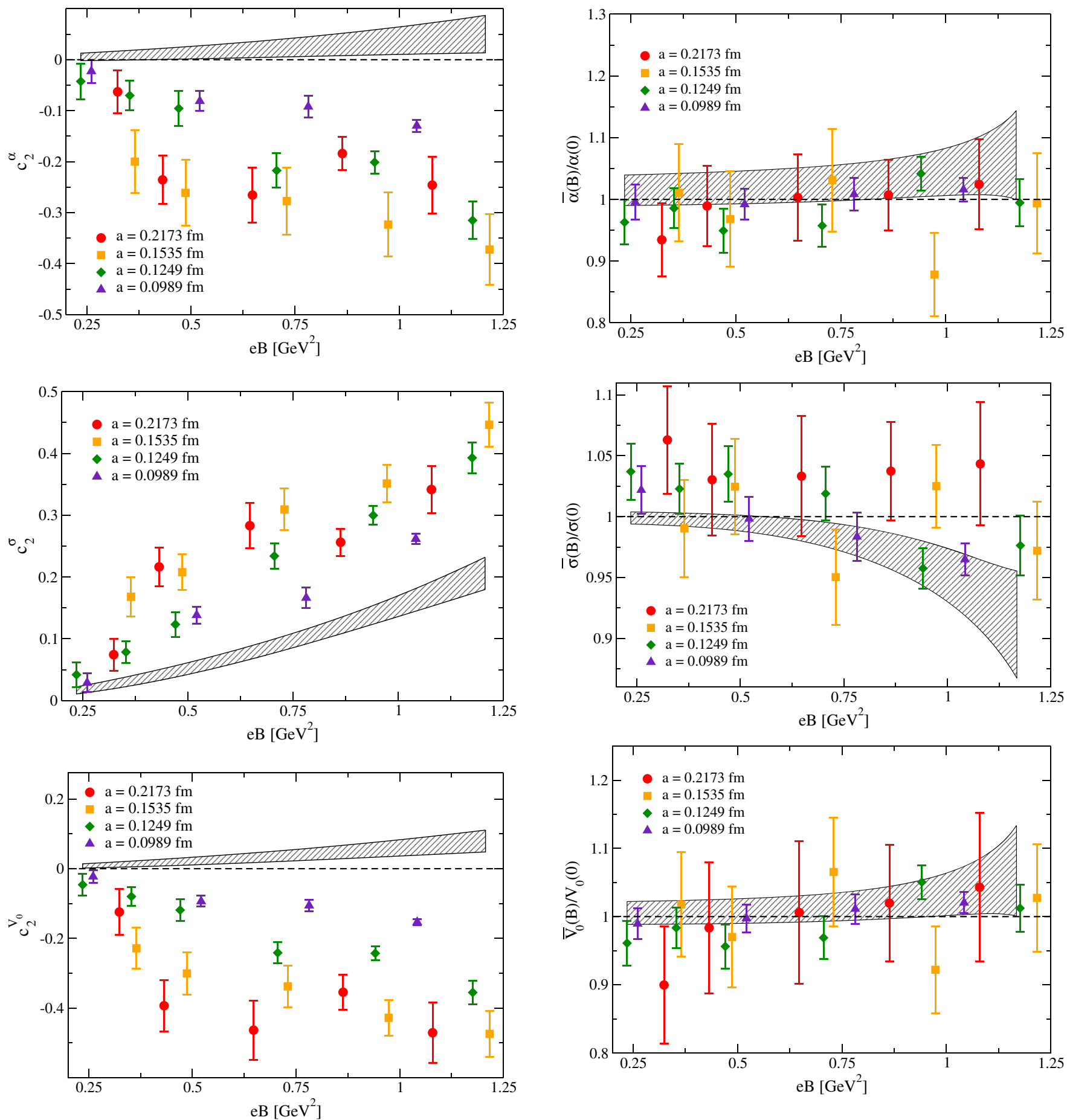

FIG. 8. The anisotropy coefficients $c_{2}^{\mathcal{O}}$ for all the potential parameters $\mathcal{O}=\alpha, \sigma, V_{0}$. Data have been computed according to the definition in Eq. (14). Gray bands represent the continuum extrapolation obtained by fitting with Eq. (18) all data except that of the coarsest lattice $24^{4}$.

signs of such anisotropies are always consistent with the fact that, at fixed $r$, the potential has a minimum (maximum) for $\theta=0(\pi / 2)$.

We performed a continuum limit extrapolation of our data according to the following ansatz:

FIG. 9. The ratios $R^{\mathcal{O}}$ for all the potential parameters $\mathcal{O}=\alpha, \sigma$, $V_{0}$. Data have been computed according to the definition in Eq. (17). Gray bands represent the continuum extrapolation obtained by fitting with Eq. (18) all data except that of the coarsest lattice $24^{4}$.

$$
\begin{aligned}
& c_{2}^{\mathcal{O}}=A^{\mathcal{O}}\left(1+C^{\mathcal{O}} a^{2}\right)(|e| B)^{D^{\mathcal{O}}\left(1+E^{\mathcal{O}} a^{2}\right)} \\
& R^{\mathcal{O}}=1+\bar{A}^{\mathcal{O}}\left(1+\bar{C}^{\mathcal{O}} a^{2}\right)(|e| B)^{\bar{D}^{\mathcal{O}}}
\end{aligned}
$$

which is similar to the one adopted in Ref. [21] and consists of a power law in $B$ for both set of quantities, with the insertion of 
MAGNETIC FIELD EFFECTS ON THE STATIC QUARK ...

$O\left(a^{2}\right)$ corrections for all involved coefficients. Reasonable fits are obtained once the data on the coarsest lattice are discarded; indeed, for the lattice spacing $a=0.2173 \mathrm{fm}$, large cutoff effects are expected for $|e| B \gtrsim 1 / a^{2} \simeq$ $0.8 \mathrm{GeV}^{2}$. Continuum extrapolated results are displayed in Fig. 8 and Fig. 9, while numerical results for the best-fit parameters are reported in Table IV. It has to be explicitly remarked that the functions in Eq. (18) are not intended to have any intrinsic physical meaning: the only physical requirements are $c_{2}^{\mathcal{O}}(B=0)=0$ and $R^{\mathcal{O}}(B=0)=1$. This form is used because it is the simplest expression that well describes data in the whole range of lattice spacing studied without introducing any specific theoretical bias. As a matter of fact, a simple linear behavior in $B$ (i.e. $D_{\mathcal{O}}=0$ and $\bar{D}_{\mathcal{O}=0}$ ) describes data almost as well and leads to continuum values which are compatible, within errors, in the explored range. We have also tried to repeat our fits on restricted ranges of $|e| B$, obtaining variations of the fitting parameters which are well within the statistical uncertainties.

The results obtained for the $c_{2}$ coefficients show that, while the anisotropy of the string tension has a well defined nonzero continuum limit, with a value around $15 \%$ for $|e| B \sim 1 \mathrm{GeV}^{2}$, those of the Coulomb coupling and of $V_{0}$ seem to disappear in the same limit. Indeed, reasonable best fits are obtained also by imposing $c_{2}^{\alpha}$ and $c_{2}^{V_{0}}$ to vanish in the continuum limit $\left(\chi^{2} / n_{\text {dof }} \sim 9.4 / 12\right.$ and $\chi^{2} / n_{\text {dof }} \simeq$ $14.4 / 12$ respectively for $c_{2}^{\alpha}$ and $c_{2}^{V_{0}}$ ), while the same is not true for $c_{2}^{\sigma}\left(\chi^{2} / n_{\mathrm{dof}} \simeq 87 / 12\right)$. The continuum extrapolated results obtained for the $R^{\mathcal{O}}$ ratios confirm, instead, that on average all Cornell parameters have little dependence on the magnetic field. Again, reasonable best fits are obtained also by imposing $R^{\mathcal{O}}$ to be $B$-independent in the continuum limit $\left(\chi^{2} / n_{\text {dof }}=8.3 / 12\right.$ for $\bar{\alpha}, \chi^{2} / n_{\text {dof }}=$ $9.1 / 12$ for $\bar{V}_{0}$, and $\chi^{2} / n_{\text {dof }}=18 / 12$ for $\bar{\sigma}$ ).

In summary, the modification of the quark-antiquark potential induced by the magnetic field persists in the continuum limit, and this is mostly due to the anisotropy induced in the string tension. In order to directly compare with the analysis of Ref. [21], in Fig. 10 we report also results for the relative variation of the string tension along the $X Y$ and $Z$ axis, together with their continuum extrapolations, obtained using the same functional form as for $R^{\mathcal{O}}$.

\section{Anisotropic deconfinement in the large field limit?}

The continuum extrapolation discussed in the previous subsection has been obtained for a range of magnetic fields

TABLE IV. Continuum limit of the anisotropies defined in Eq. (14), performed using the ansatz in Eq. (18). The fit does not include the coarsest $24^{4}$ lattice.

\begin{tabular}{llllll}
\hline \hline$A^{\sigma}$ & $0.151(32)$ & $D^{\sigma}$ & $1.64(30)$ & $\chi^{2} / n_{\text {dof }}$ & $9.5 / 11$ \\
\hline$A^{\alpha}$ & $0.046(39)$ & $D^{\alpha}$ & $1.51(67)$ & $\chi^{2} / n_{\text {dof }}$ & $7.3 / 11$ \\
$A^{V_{0}}$ & $0.066(36)$ & $D^{V_{0}}$ & $1.48(50)$ & $\chi^{2} / n_{\text {dof }}$ & $7.8 / 11$ \\
\hline \hline
\end{tabular}

PHYSICAL REVIEW D 94, 094007 (2016)

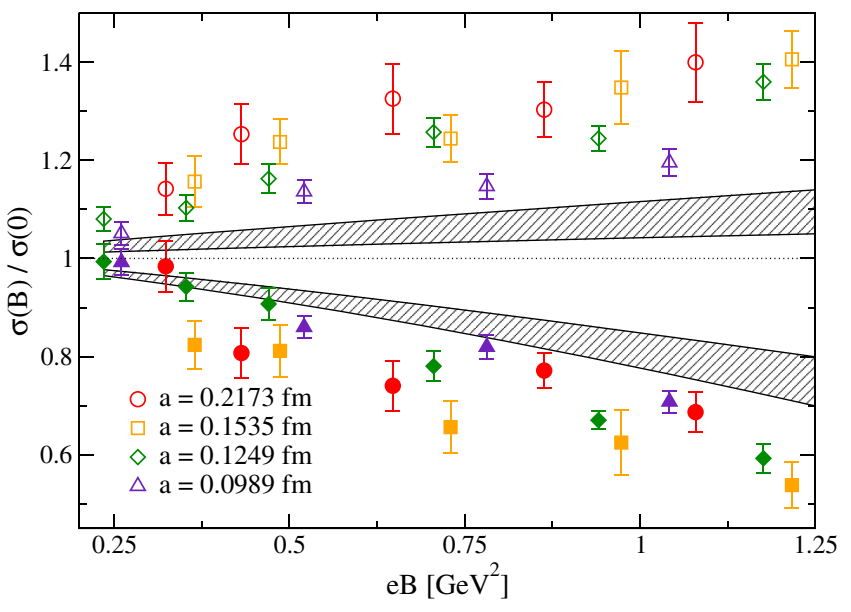

FIG. 10. Ratios between the string tension computed along the $X Y$ (empty symbols) and the $Z$ (full symbol) directions and the string tension computed at $B=0$. Bands denote the continuum extrapolations, which have been obtained using an ansatz analogous to the one used in Eq. (18) on the three finest lattice spacings. Best-fit parameters (relevant in the continuum limit) are $A^{\sigma_{X Y}}=0.084(42), D^{\sigma_{X Y}}=0.90(37)$, with $\chi^{2} / n_{\text {dof }}=8.9 / 11$, and $A^{\sigma_{Z}}=-0.198(39), D^{\sigma_{Z}}=2.08(44)$ with $\chi^{2}=10.6 / 11$.

going up to around $1 \mathrm{GeV}^{2}$; however, it is tempting to extrapolate it to larger values of $B$. In particular, results obtained for the longitudinal string tension indicate that it would vanish for magnetic fields of the order of $4 \mathrm{GeV}^{2}$ : this is clearly visible from Fig. 11, where the continuum extrapolation for the ratio $\sigma(|e| B) / \sigma(0)$ along the axes has been extended up to $e B \sim 4 \mathrm{GeV}^{2}$.

A vanishing string tension at some critical value of $B$, corresponding to a sort of longitudinal deconfinement, is compatible with large- $B$ effective field theories (see, e.g., Ref. [100]) and would have important consequences, e.g.,

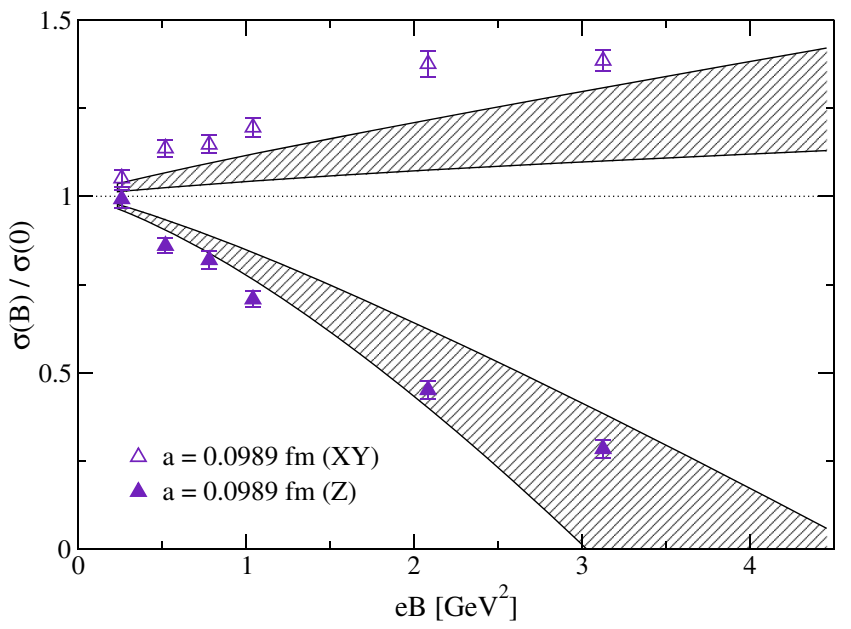

FIG. 11. Relative change of the string tension as a function of $|e| B$, along the directions parallel and orthogonal to the background field. The shadowed band corresponds to the large field extrapolation of the continuum limit obtained for $|e| B \lesssim 1 \mathrm{GeV}^{2}$. 
for the anisotropic propagation of heavy quark-antiquark pairs, and could be put in connection with theoretical speculations about anisotropic quantum transitions at large values of $B$ [101].

A question naturally arises at this point: how reliable is the extension to large magnetic fields of a continuum extrapolation obtained in a smaller range of $B$ ? In order to better appreciate the question, we stress that assumptions different from Eq. (18) could lead to different predictions in the large $B$ region: for instance, if a linear behavior in $B$ is assumed, the continuum limit does not change within errors for $e|B| \lesssim 1 \mathrm{GeV}^{2}$, but the point where the longitudinal string tension vanishes moves to $e|B| \sim 10 \mathrm{GeV}^{2}$.

Unfortunately, a direct continuum extrapolation for $|e| B \gg 1 \mathrm{GeV}^{2}$ is presently hindered by the large ultraviolet cutoff effects which are expected when $|e| B \gtrsim 1 / a^{2}$ [74]. This sets the limit $|e| B \lesssim 1 \mathrm{GeV}^{2}$ for two of the lattice spacings explored in the present study.

To have more reliable indications of what happens at such large fields, we can extend the range of explored $|e| B$ just for the finest lattice spacing, $a \simeq 0.0989 \mathrm{fm}$, for which $1 / a^{2} \sim 4 \mathrm{GeV}^{2}$. Therefore, we have performed further numerical simulations for $|e| B \sim 2$ and $3 \mathrm{GeV}^{2}$, obtaining the results for the string tension which are reported in Fig. 11. The new results suggest that the steady decrease of the longitudinal string tension persists up to very large fields, even if with a slight tendency to undershoot the extrapolation of the continuum band, so that its vanishing at some critical magnetic field is a concrete possibility. On the other hand, understanding whether an anisotropic deconfinement really takes place at some critical value of $B$ requires further dedicated simulations at finer values of the lattice spacing.

\section{E. $T>0$ results}

For finite temperature runs, we have extracted the static potential from the gauge invariant correlator of Polyakov loops, see Eqs. (6) and (7). In this case we report on results at a single value of the lattice spacing (the finest one, $a \simeq 0.0989 \mathrm{fm}$ ) and for three temperatures below the transition temperature, namely $T \simeq 100,125,143 \mathrm{MeV}$ (corresponding to lattices $48^{3} \times 20,16,14$, respectively), where we still expect the system may exhibit confining properties. In the deconfined phase, instead, Polyakov loop correlators give access to various kind of gluonic screening masses: the effect of the magnetic field on those masses will be studied in a forthcoming investigation. In Fig. 12 we show the results obtained for $F_{Q \bar{Q}}$ at some values of the magnetic field, respectively for $T \simeq 100 \mathrm{MeV}$ and $T \simeq 125 \mathrm{MeV}$. In general, we observe a behavior which is different from that present at $T=0$.

The anisotropy is still visible, and goes in the same direction as for $T=0$, with the potential for quarkantiquark separations parallel to $\vec{B}$ being suppressed with
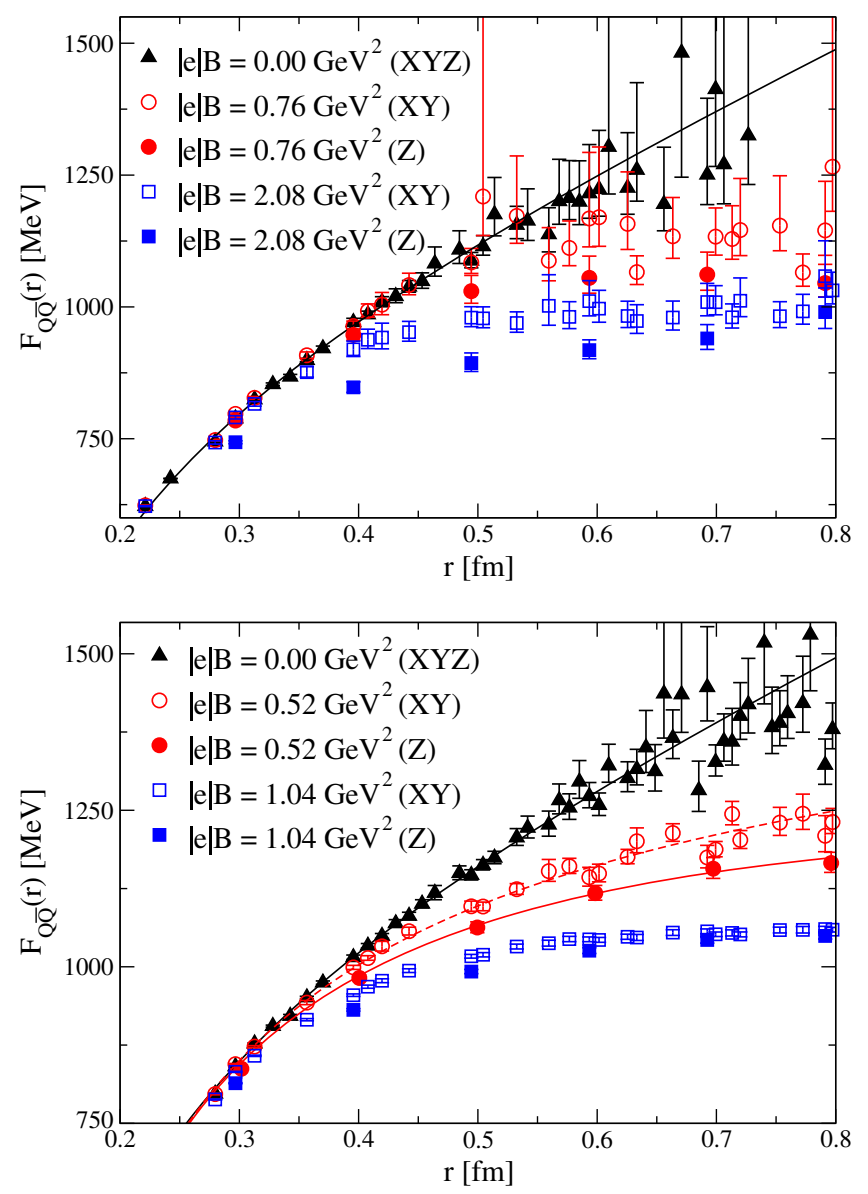

FIG. 12. Top: Free energy of the $Q \bar{Q}$ pair $F_{Q \bar{Q}}(r, T)$ at $T \simeq 100 \mathrm{MeV}\left(48^{3} \times 20\right.$ lattice $)$ as a function of the distance $r$ for several values of $B$. Bottom: $F_{Q \bar{Q}}(r, T)$ at $T \simeq 125 \mathrm{MeV}$ $\left(48^{3} \times 16\right.$ lattice). Continuum and dashed curves correspond to best fits to a Cornell potential, and are reported only for cases in which the best fit works well in a reasonable range of distances.

respect to the orthogonal case. The anisotropy is more pronounced for large magnetic fields and for intermediate separations, while it disappears in the limit of large distances: this is actually expected, since the magnetic background cannot disrupt the cluster property of the theory; hence, the correlation of two Polyakov loops must be independent, in the large distance limit, from the direction of their relative separation.

Contrary to the zero temperature case, the main effect of the magnetic field seems to be that of suppressing the potential in all directions. Only for relatively small magnetic fields (smaller and smaller as the temperature increases) can the observed $F_{Q \bar{Q}}$ be fitted according to a Cornell potential and is qualitatively similar to that observed for $T=0$. However, in these cases one is not able to distinguish, within errors, the results obtained in the various directions: this is due to the larger statistical uncertainties affecting the Polyakov loop correlator, especially in the low temperature regime, where the Polyakov 
loop is more suppressed and the correlators are noisier. The results obtained for the string tension in such cases are reported in Fig. 13. As expected for vanishing $|e| B$, the string tension $\sigma$ decreases as the temperature approaches the deconfinement transition. Such an effect comes out to be enhanced when the external field is turned on.

The observed behavior is consistent with the decrease of the chiral pseudocritical temperature which has been observed in previous studies $[15,17]$. This phenomenon has been usually named as inverse magnetic catalysis, because of its relation with the behavior of the chiral condensate, which is an increasing function of $B$ at low $T$ (direct magnetic catalysis), and becomes nonmonotonic around the transition, in association with a decrease of $T_{c}$. Our observations provide evidence for a strong effect of the magnetic field also on the confining properties of the medium which, at fixed temperature, seem to be lost if the magnetic field is strong enough.

Actually, looking at the behavior of the chiral condensate for the same values of temperature and magnetic field, the effect on the confining properties seems to be the leading phenomenon. In Fig. 14 we report the renormalized light chiral condensate as a function of $B$ for the three explored temperatures, which is defined as [15]

$$
\langle\bar{\psi} \psi\rangle^{r}(T, B)=\frac{m_{l}}{m_{\pi}^{4}}\left(\langle\bar{\psi} \psi\rangle_{l}(T, B)-\langle\bar{\psi} \psi\rangle_{l}(0,0)\right)
$$

where the $T=0$ subtraction eliminates additive divergences, while the multiplication by the bare light quark mass $m_{l}$ takes care of multiplicative ones. It is clearly visible that for the two lowest temperatures, no signal of inverse magnetic catalysis is visible in the explored range of $B$, while in the same range we already observe a strong modification of the free energy of the static pair, such that

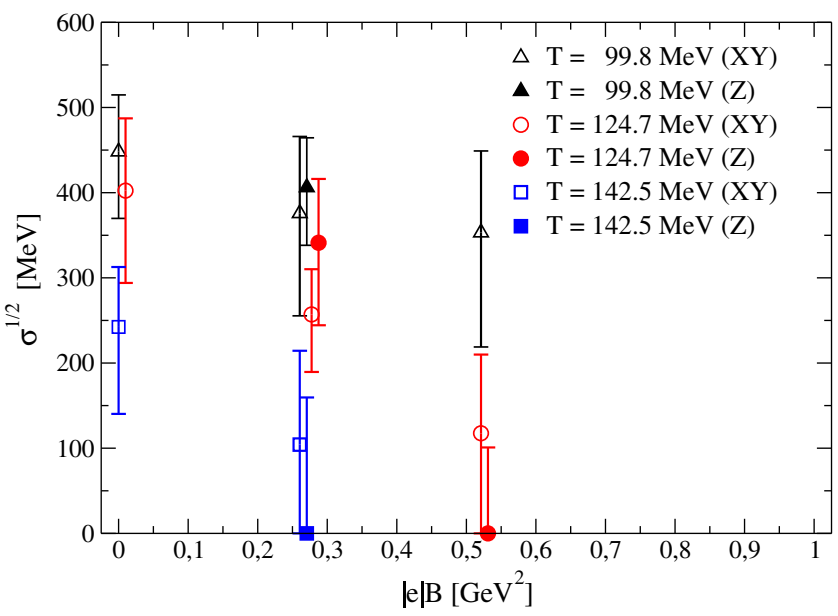

FIG. 13. The square root of the string tension $\sigma$ along different directions is reported, as a function of $|e| B$, for three different temperatures below $T_{c}$ and for $a=0.0989 \mathrm{fm}$. In the case $|e| B=$ 0 data refer to an average over all directions.

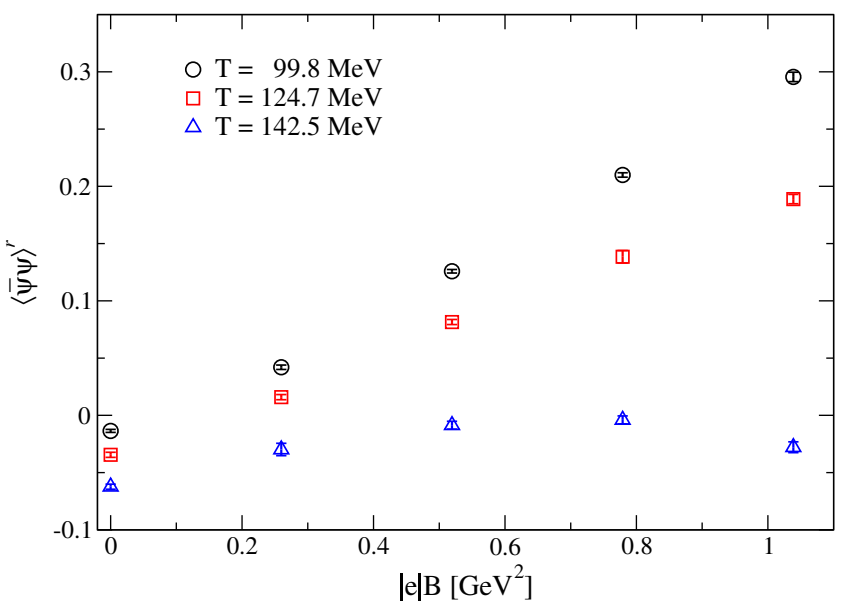

FIG. 14. Renormalized chiral condensate as a function of the magnetic field for various temperatures.

we are not able to fit it according to a Cornell potential, something which we interpret as an effective disappearance of the confining properties.

This might have many interesting interpretations and consequences. First, from a theoretical point of view, the suppression of the confining properties seems to be a dominant phenomenon with respect to the effect of the magnetic field on the chiral condensate, so that one would be tempted to describe the observed decrease of $T_{c}$ with $B$ in terms of "deconfinement catalysis" rather than of "inverse magnetic catalysis"; notice that this point of view is also suggested by recent computations in effective models [45,46] and holographic computations [49]. Second, from a phenomenological point of view, the precocious modification of the confining properties induced by the magnetic background might have significant consequences on the suppression of heavy quark bound states (e.g., $J / \psi$ ) to be observed in the thermal medium produced in noncentral heavy ion collisions.

\section{CONCLUSIONS}

Following an explorative study [21], which showed the presence of strong effects on the confining properties of the QCD vacuum induced by the presence of an external magnetic background, we have performed a deeper investigation of the phenomenon, which has extended the previous analysis of Ref. [21] in various directions. We have thus studied the static potential for various orientations of the quark-antiquark separation with respect to the background field, in order to obtain information about its angular dependence. Then, exploiting new numerical simulations performed for finer values of the lattice spacing, we have extracted a continuum extrapolation of our results and tried to extend the analysis towards larger magnetic fields. As a byproduct of our study, we have also obtained a continuum extrapolation of the static potential at 
zero external field. Finally, we have extended our investigation at finite temperature, exploring the effects of the magnetic background below the pseudocritical temperature $T_{c}$. Our main results can be summarized as follows.

At zero temperature, the full angular dependence of the anisotropic potential can be described assuming that it has a Cornell form along each direction. Moreover, the angular dependence of the parameters can be accounted for by the first allowed term $c_{2}$ in a Fourier expansion in $\theta$, which corresponds to a quadrupole deformation. On the other hand, the continuum extrapolation of our results shows that the magnetic field induces a significant anisotropy only for the string tension, while the Coulomb coupling is almost unaffected, at least for $|e| B \lesssim 1 \mathrm{GeV}^{2}$. The latter result is compatible with an analysis based on the numerical study of gluon field strength correlators [23].

The observed anisotropy of the potential suggests that the string tension in the direction parallel to the magnetic field might disappear for magnetic fields of the order of $|e| B \sim 4 \mathrm{GeV}^{2}$. While this extrapolation to large magnetic fields cannot be supported by a reliable continuum limit in that regime, we have verified that it is consistent with results obtained at the finest explored lattice spacing, where we managed to perform numerical simulations for magnetic fields up $|e| B \sim 3 \mathrm{GeV}^{2}$. Future numerical studies on finer lattices could check the hypothesis of a possible longitudinal deconfinement at large $B$, which presently is just suggested by our results.

Finally, our finite temperature results, obtained in an interval ranging approximately from $100 \mathrm{MeV}$ to right below the pseudocritical temperature, have shown that the main effect of the magnetic field on the static potential in that range consists of a general suppression of the string tension and of the confining properties of the medium. Moreover, such a phenomenon still happens even when no signal of inverse magnetic catalysis is visible in the chiral condensate, thus suggesting that the decrease of the pseudocritical temperature as a function of $B$, usually named as "inverse magnetic catalysis," might accompany and follow a "deconfinement catalysis," representing the leading physical phenomenon: this idea is also supported by some recent computations in effective models [45,46] and holographic computations [49]. From a phenomenological point of view, the precocious disappearance of the confining properties of the static potential might have a significant influence on the suppression of heavy quark bound states produced in the thermal medium, even below $T_{c}$, in all situations in which a strong magnetic field might be present, e.g., in noncentral heavy ion collisions and in the thermal medium of the early Universe. This is an issue of particular interest and should be further investigated in future studies.

\section{ACKNOWLEDGMENTS}

We acknowledge PRACE for awarding us access to resource FERMI based in Italy at CINECA, under Project No. Pra09-2400-SISMAF. F. S. received funding from the European Research Council under the European Community Seventh Framework Programme (FP7/20072013) ERC Grant No. 279757. F. N. acknowledges financial support from the INFN SUMA project.
[1] V. Skokov, A. Y. Illarionov, and V. Toneev, Int. J. Mod. Phys. A 24, 5925 (2009).

[2] V. Voronyuk, V. D. Toneev, W. Cassing, E. L. Bratkovskaya, V. P. Konchakovski, and S. A. Voloshin, Phys. Rev. C 83, 054911 (2011).

[3] A. Bzdak and V. Skokov, Phys. Lett. B 710, 171 (2012).

[4] W.-T. Deng and X.-G. Huang, Phys. Rev. C 85, 044907 (2012).

[5] K. Tuchin, Adv. High Energy Phys. 2013, 490495 (2013).

[6] R. Holliday and K. Tuchin, arXiv:1604.04572.

[7] T. Vachaspati, Phys. Lett. B 265, 258 (1991).

[8] D. Grasso and H. R. Rubinstein, Phys. Rep. 348, 163 (2001).

[9] R. C. Duncan and C. Thompson, Astrophys. J. 392, L9 (1992).

[10] D. E. Kharzeev, K. Landsteiner, A. Schmitt, and H. U. Yee, Lect. Notes Phys. 871, 1 (2013).

[11] V. A. Miransky and I. A. Shovkovy, Phys. Rep. 576, 1 (2015).
[12] M. D'Elia, S. Mukherjee, and F. Sanfilippo, Phys. Rev. D 82, 051501 (2010).

[13] P. V. Buividovich, M. N. Chernodub, D. E. Kharzeev, T. Kalaydzhyan, E. V. Luschevskaya, and M. I. Polikarpov, Phys. Rev. Lett. 105, 132001 (2010).

[14] M. D’Elia and F. Negro, Phys. Rev. D 83, 114028 (2011).

[15] G. S. Bali, F. Bruckmann, G. Endrodi, Z. Fodor, S. D. Katz, S. Krieg, A. Schafer, and K. K. Szabo, J. High Energy Phys. 02 (2012) 044.

[16] E.-M. Ilgenfritz, M. Kalinowski, M. Muller-Preussker, B. Petersson, and A. Schreiber, Phys. Rev. D 85, 114504 (2012).

[17] G. S. Bali, F. Bruckmann, G. Endrodi, Z. Fodor, S. D. Katz, and A. Schafer, Phys. Rev. D 86, 071502 (2012).

[18] M. D'Elia, M. Mariti, and F. Negro, Phys. Rev. Lett. 110, 082002 (2013).

[19] F. Bruckmann, G. Endrodi, and T. G. Kovacs, J. High Energy Phys. 04 (2013) 112

[20] E.-M. Ilgenfritz, M. Muller-Preussker, B. Petersson, and A. Schreiber, Phys. Rev. D 89, 054512 (2014). 
[21] C. Bonati, M. D’Elia, M. Mariti, M. Mesiti, F. Negro, and F. Sanfilippo, Phys. Rev. D 89, 114502 (2014).

[22] A. Schäfer, G. Endrodi, and J. Wellnhofer, Phys. Rev. D 92, 014509 (2015).

[23] M. D’Elia, E. Meggiolaro, M. Mesiti, and F. Negro, Phys. Rev. D 93, 054017 (2016).

[24] C. Bonati, M. D'Elia, M. Mariti, F. Negro, and F. Sanfilippo, Phys. Rev. D 89, 054506 (2014); Phys. Rev. Lett. 111, 182001 (2013).

[25] L. Levkova and C. DeTar, Phys. Rev. Lett. 112, 012002 (2014).

[26] G. S. Bali, F. Bruckmann, G. Endrodi, F. Gruber, and A. Schaefer, J. High Energy Phys. 04 (2013) 130.

[27] G. S. Bali, F. Bruckmann, G. Endrodi, S. D. Katz, and A. Schaefer, J. High Energy Phys. 08 (2014) 177.

[28] P. Cea and L. Cosmai, J. High Energy Phys. 12 (2015) 058.

[29] M. M. Musakhanov and F. C. Khanna, arXiv:hep-ph/ 9605232.

[30] J. Rafelski, in Proceedings of the Workshop on Frontier Tests of Quantum Electrodynamics and Physics of the Vacuum, Sandansky, Bulgaria, edited by E. Zavattini, D. Bakalov, and C. Rizzo (Heron Press, Sofia, 1998).

[31] H. T. Elze, B. Muller, and J. Rafelski, arXiv:hep-ph/ 9811372.

[32] M. N. Chernodub, arXiv:1001.0570.

[33] M. Asakawa, A. Majumder, and B. Muller, Phys. Rev. C 81, 064912 (2010).

[34] B. V. Galilo and S. N. Nedelko, Phys. Rev. D 84, 094017 (2011).

[35] M. A. Andreichikov, V. D. Orlovsky, and Y. A. Simonov, Phys. Rev. Lett. 110, 162002 (2013).

[36] V. D. Orlovsky and Y. A. Simonov, Phys. Rev. D 89, 054012 (2014).

[37] T. Kojo and N. Su, Phys. Lett. B 720, 192 (2013).

[38] T. Kojo and N. Su, Phys. Lett. B 726, 839 (2013).

[39] P. Watson and H. Reinhardt, Phys. Rev. D 89, 045008 (2014).

[40] J. O. Andersen, W. R. Naylor, and A. Tranberg, J. High Energy Phys. 04 (2014) 187.

[41] S. Ozaki, Phys. Rev. D 89, 054022 (2014).

[42] K. Kamikado and T. Kanazawa, J. High Energy Phys. 03 (2014) 009.

[43] N. Mueller, J. A. Bonnet, and C. S. Fischer, Phys. Rev. D 89, 094023 (2014).

[44] N. Mueller and J. M. Pawlowski, Phys. Rev. D 91, 116010 (2015).

[45] E. S. Fraga and L. F. Palhares, Phys. Rev. D 86, 016008 (2012).

[46] E. S. Fraga, J. Noronha, and L. F. Palhares, Phys. Rev. D 87, 114014 (2013).

[47] K. A. Mamo, J. High Energy Phys. 05 (2015) 121.

[48] R. Rougemont, R. Critelli, and J. Noronha, Phys. Rev. D 93, 045013 (2016).

[49] D. Dudal, D. R. Granado, and T. G. Mertens, Phys. Rev. D 93, 125004 (2016).

[50] R. L. S. Farias, V. S. Timoteo, S. S. Avancini, M. B. Pinto, and G. Krein, arXiv:1603.03847.

[51] S. I. Finazzo, R. Critelli, R. Rougemont, and J. Noronha, Phys. Rev. D 94, 054020 (2016).

[52] B. McInnes, Nucl. Phys. B906, 40 (2016).
[53] V. A. Miransky and I. A. Shovkovy, Phys. Rev. D 66, 045006 (2002).

[54] M. N. Chernodub, Mod. Phys. Lett. A 29, 1450162 (2014).

[55] R. Rougemont, R. Critelli, and J. Noronha, Phys. Rev. D 91, 066001 (2015).

[56] E. J. Ferrer, V. de la Incera, and X. J. Wen, Phys. Rev. D 91, 054006 (2015).

[57] Y. A. Simonov and M. A. Trusov, Phys. Lett. B 747, 48 (2015).

[58] J. Alford and M. Strickland, Phys. Rev. D 88, 105017 (2013).

[59] D. Dudal and T. G. Mertens, Phys. Rev. D 91, 086002 (2015).

[60] S. Cho, K. Hattori, S. H. Lee, K. Morita, and S. Ozaki, Phys. Rev. D 91, 045025 (2015).

[61] H. Taya, Phys. Rev. D 92, 014038 (2015).

[62] C. Bonati, M. D’Elia, and A. Rucci, Phys. Rev. D 92, 054014 (2015).

[63] K. Hattori, T. Kojo, and N. Su, Nucl. Phys. A951, 1 (2016).

[64] K. Suzuki and T. Yoshida, Phys. Rev. D 93, 051502 (2016); arXiv:1607.04935 [to be published (Phys. Rev. D)].

[65] X. Guo, S. Shi, N. Xu, Z. Xu, and P. Zhuang, Phys. Lett. B 751, 215 (2015).

[66] K. Fukushima, K. Hattori, H. U. Yee, and Y. Yin, Phys. Rev. D 93, 074028 (2016).

[67] P. Gubler, K. Hattori, S. H. Lee, M. Oka, S. Ozaki, and K. Suzuki, Phys. Rev. D 93, 054026 (2016).

[68] P. Weisz, Nucl. Phys. B212, 1 (1983).

[69] G. Curci, P. Menotti, and G. Paffuti, Phys. Lett. 130B, 205 (1983); 135B, 516(E) (1984).

[70] C. Morningstar and M. J. Peardon, Phys. Rev. D 69, 054501 (2004).

[71] G.'t Hooft, Nucl. Phys. B153, 141 (1979).

[72] P. H. Damgaard and U. M. Heller, Nucl. Phys. B309, 625 (1988).

[73] M. H. Al-Hashimi and U.J. Wiese, Ann. Phys. (Amsterdam) 324, 343 (2009).

[74] M. D’Elia, Lect. Notes Phys. 871, 181 (2013).

[75] Y. Aoki, S. Borsanyi, S. Durr, Z. Fodor, S. D. Katz, S. Krieg, and K. K. Szabo, J. High Energy Phys. 06 (2009) 088.

[76] S. Borsanyi, G. Endrodi, Z. Fodor, A. Jakovac, S. D. Katz, S. Krieg, C. Ratti, and K. K. Szabo, J. High Energy Phys. 11 (2010) 077.

[77] S. Borsanyi, Z. Fodor, C. Hoelbling, S. D. Katz, S. Krieg, and K. K. Szabo, Phys. Lett. B 730, 99 (2014).

[78] M. A. Clark, A. D. Kennedy, and Z. Sroczynski, Nucl. Phys. B, Proc. Suppl. 140, 835 (2005).

[79] M. A. Clark and A. D. Kennedy, Phys. Rev. Lett. 98, 051601 (2007).

[80] M. A. Clark and A. D. Kennedy, Phys. Rev. D 75, 011502 (2007).

[81] A. Hasenfratz and F. Knechtli, Phys. Rev. D 64, 034504 (2001).

[82] M. Albanese et al. (APE Collaboration), Phys. Lett. B 192, 163 (1987).

[83] M. Della Morte, A. Shindler, and R. Sommer, J. High Energy Phys. 08 (2005) 051.

[84] L. S. Brown and W. I. Weisberger, Phys. Rev. D 20, 3239 (1979). 
[85] L. D. McLerran and B. Svetitsky, Phys. Rev. D 24, 450 (1981).

[86] S. Nadkarni, Phys. Rev. D 34, 3904 (1986).

[87] O. Kaczmarek, F. Karsch, P. Petreczky, and F. Zantow, Phys. Lett. B 543, 41 (2002).

[88] O. Jahn and O. Philipsen, Phys. Rev. D 70, 074504 (2004).

[89] G. Rossi and M. Testa, Phys. Rev. D 87, 085014 (2013).

[90] E. Eichten, K. Gottfried, T. Kinoshita, J. B. Kogut, K. D. Lane, and T. M. Yan, Phys. Rev. Lett. 34, 369 (1975); 36, 1276(E) (1976).

[91] R. Sommer, Nucl. Phys. B411, 839 (1994).

[92] B. Blossier et al. (ETM Collaboration), J. High Energy Phys. 07 (2009) 043.

[93] R. Baron et al. (ETM Collaboration), J. High Energy Phys. 08 (2010) 097.
[94] P. Fritzsch, F. Knechtli, B. Leder, M. Marinkovic, S. Schaefer, R. Sommer, and F. Virotta, Nucl. Phys. B865, 397 (2012).

[95] G. S. Bali et al., Nucl. Phys. B866, 1 (2013).

[96] C. T. H. Davies, E. Follana, I. D. Kendall, G. Peter Lepage, and C. McNeile (HPQCD Collaboration), Phys. Rev. D 81, 034506 (2010).

[97] A. Bazavov et al., Phys. Rev. D 85, 054503 (2012).

[98] C. Aubin, C. Bernard, C. DeTar, J. Osborn, S. Gottlieb, E. B. Gregory, D. Toussaint, U. M. Heller, J. E. Hetrick, and R. Sugar, Phys. Rev. D 70, 094505 (2004).

[99] S. Aoki et al., arXiv:1607.00299.

[100] G. Endrodi, J. High Energy Phys. 07 (2015) 173.

[101] M. N. Chernodub, Phys. Rev. Lett. 106, 142003 (2011); Phys. Rev. D 82, 085011 (2010). 